\title{
GIS BASED MAPPING OF SAFE BEARING CAPACITY FOR THE STATE OF GOA
}

\author{
Subash Goudar \\ Post Graduate Student, ME Foundation Engineering GEC, \\ Farmagudi Goa - 403401 \\ Nisha P. Naik \\ Associate Professor Department of Civil Engineering GEC, \\ Farmagudi Goa - 403401
}

\begin{abstract}
Soil is the most important yet neglected factor in the construction projects. Consumers see soil analysis as a fund loss despite being the cheapest in the construction process. Some contractors also ignore the importance of proper soil research and analysis and base their design on assumed bearing capacity and settlement rate. The present paper is based on mapping of SBC (Safe Bearing Capacity) of soil. In this work, GIS based map of SBC has been developed for the state of Goa. Data around 200 Borelogs was collected from different agencies. The necessary data was extracted from borelogs and calculation and mapping of SBC carried out. These maps will help the engineers and contractors to get a preliminary idea of the soil strata and serve as a guideline for the design the foundations and also to carry out other construction works.
\end{abstract}

Keywords: GIS, GPS, SBC, Geotechnical engineering.

\section{INTRODUCTION}

The soil is a characteristic material with a variety of physical and mechanical characteristics, the vast majority of which are not steady and differ from place to place. Bearing capacity is the capacity of soil to support the loads applied to the ground. The bearing capacity of soil is the maximum average contact pressure between the foundation and the soil which should not produce shear failure in the soil (Ramamurthy \& Sitharam 2005). Most of the work that was referred included the mapping soil properties. In this work an attempt was done to map SBC of soil. By mapping the SBC for Goa, it will be very useful for the contractors, geotechnical engineers to predict and suggest the desired foundation and necessary precautions to be taken for improvement of soil and its properties.

\section{LITERATURE REVIEW}

A foundation is the part of a structure which transmits the weight of the structure to the ground. All structures constructed on land are supported on foundations. A foundation is a connecting link between the structure and the ground which supports it. The bearing strength characteristics of foundation soil are major design criterion for civil engineering structures. In non-technical engineering, bearing capacity is the capacity of soil to support the loads applied to the ground. The bearing capacity of soil is the maximum average contact pressure between the foundation and the soil which should not produce shear failure in the soil. Ultimate bearing capacity is the theoretical maximum pressure which can be supported without failure; allowable bearing capacity is the ultimate bearing capacity divided by a factor of safety. Sometimes, on soft soil sites, large settlements may occur under loaded foundations without actual shear failure occurring; in such cases, the allowable bearing capacity is based on the maximum allowable settlement (B.C. Punmia, 2005)

Mhaske et al. (2009) generated thematic maps (Figure 1) of soil properties such as moisture content, specific gravity, liquid limit, plastic limit, etc. using GRAM++ software of some places for Mumbai city. M. L. Manchanda et al. (2002) generated maps of Doon valley and came to conclusion that for precise soil mapping, larger amount of data is required. T.G. Sitharam et al. (2005) generated maps of peak ground acceleration and the factor of safety against liquefaction maps for Bangalore city. Md. M Rahman \& A. Q. M. Mahabub (2012) generated maps to identify the location of aquifer at Barind Tract in 


\section{International Journal of Engineering Applied Sciences and Technology, 2020 \\ Vol. 4, Issue 10, ISSN No. 2455-2143, Pages 186-195 \\ Published Online February 2020 in IJEAST (http://www.ijeast.com)}

northwest Bangladesh. M. Labib and A. Nashed (2013) generated maps of expansive soils along Sheikh Zayed canal.

No maps of SBC were generated for the state of Goa is found in literature.

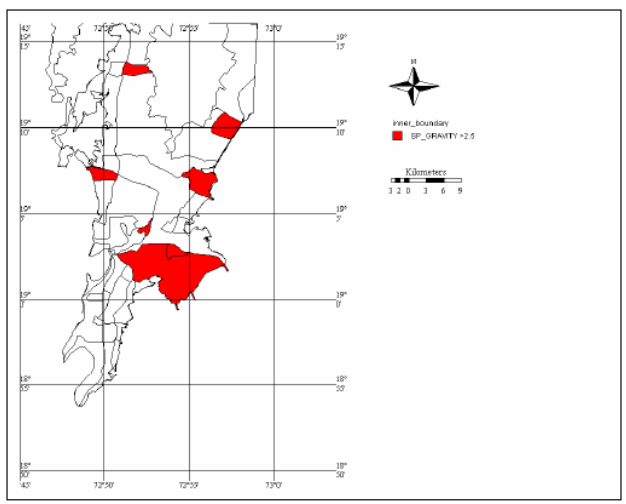

Figure 1 Thematic map for specific gravity of typical soil in Mumbai city (source: Mhaske S.Y. et al, IGC, 2009)

III. METHODOLOGY
The work carried out required collection of borelogs across the state of Goa. Around 200 numbers of borelogs were collected from different agencies. The borelogs were collected from 3 soil investigation agencies, real estate developers and public works department Goa. The various properties of soil i.e. density, Atterberg's limits, cohesion, angle of friction, water table, specific gravity and soil layer were extracted from soil reports. The calculation of SBC was carried out using IS 6403 1891. The SBC was determined for two different depths (i.e. $1.5 \mathrm{~m}$ and $3 \mathrm{~m}$ ). The SBC was calculated by SPT $\mathrm{N}$ value and cohesion and angle of internal friction values. Database for SBC of soil was created from the boreholes accordingly meeting the requirements of the GIS software. Table 1, 2, 3 \& 4 shows the SBC calculation of few boreholes. Overlaying of spatial \& non spatial data was done in ArcGIS software in order to obtain the maps. Digitization of the outline of Goa was done using polygon type shapefile. Latitudes and Longitudes of the different borehole locations along with all the soil properties were input in the ArcGIS software to generate all types of maps. With the help of IDW (Inverse Distance Weighing) interpolation feature of the software all the maps were interpolated.

Table 1 SBC calculation table (by $\mathrm{N}$ value) (for $1.5 \mathrm{~m}$ )

\begin{tabular}{|c|c|c|c|c|c|c|c|c|c|c|c|c|c|}
\hline Place & Lat & Long & $\begin{array}{l}\text { SPT } \\
(\mathbf{N})\end{array}$ & $\begin{array}{l}\mathbf{W T}(\mathbf{m}) \\
\left(\mathbf{D}_{\mathbf{t}}\right)\end{array}$ & B & D & $\mathbf{Y}_{\mathbf{d}}$ & C & $\begin{array}{l}\varphi \\
\left({ }^{\circ}\right)\end{array}$ & $\mathbf{N}_{\mathbf{C}}$ & $\mathbf{N}_{\mathbf{q}}$ & $\mathbf{N}_{r}$ & $\begin{array}{l}\text { qs } \\
\left(\mathbf{k g} / \mathbf{c m}^{2}\right)\end{array}$ \\
\hline Neogi Nagar & 15.498 & 73.821 & 2 & 0.3 & 1.5 & 1.5 & 1.03 & 0 & 28 & 26.37 & 15.3 & 17.79 & 41.96 \\
\hline School at Campal & 15.495 & 73.819 & 13 & 1.3 & 1.5 & 1.5 & 1.53 & 0 & 31 & 33.33 & 21.38 & 27.52 & 112.34 \\
\hline $\begin{array}{l}\text { Tonca, Panaji } \\
\text { (Prudential groups) }\end{array}$ & 15.494 & 73.825 & 15 & 28.54 & 1.5 & 1.5 & 1.64 & 0 & 32 & 36.53 & 24.36 & 32.65 & 896.54 \\
\hline $\begin{array}{l}\text { Panaji (Hotel } \\
\text { Nova) }\end{array}$ & 15.498 & 73.823 & 4 & 0.87 & 1.5 & 1.5 & 1.34 & 0 & 29 & 28.25 & 16.85 & 20.09 & 68.81 \\
\hline $\begin{array}{l}\text { Panaji (Vistar } \\
\text { motors portais) }\end{array}$ & 15.495 & 73.825 & 1 & 1.3 & 1.5 & 1.5 & 1.52 & 0 & 28 & 26.37 & 15.3 & 17.79 & 76.21 \\
\hline Mapusa & 15.607 & 73.805 & 17 & 0.43 & 1.5 & 1.5 & 1.47 & 0 & 32 & 36.53 & 24.36 & 32.65 & 103.52 \\
\hline Ucassaim, Mapusa & 15.58 & 73.842 & 50 & 1.1 & 1.5 & 1.5 & 1.66 & 0 & 41 & 88.02 & 78.33 & 141.87 & 501.47 \\
\hline $\begin{array}{l}\text { Dry dock, } \\
\text { Bainguinim, Old } \\
\text { Goa }\end{array}$ & 15.455 & 73.814 & 1 & 1 & 1.5 & 1.5 & 1.46 & 0 & 28 & 26.37 & 15.3 & 17.79 & 69.08 \\
\hline
\end{tabular}


International Journal of Engineering Applied Sciences and Technology, 2020

Vol. 4, Issue 10, ISSN No. 2455-2143, Pages 186-195

Published Online February 2020 in IJEAST (http://www.ijeast.com)

Table 2 SBC calculation table (by $\mathrm{C} \& \varphi$ value) (for $1.5 \mathrm{~m}$ )

\begin{tabular}{|c|c|c|c|c|c|c|c|c|c|c|c|c|}
\hline Place & Lat & Long & $\mathbf{W T}(\mathbf{m})\left(\mathbf{D}_{t}\right)$ & B & D & $\mathbf{r}_{\mathrm{d}}$ & C & $\begin{array}{l}\varphi \\
\left(^{\circ}\right)\end{array}$ & $\mathbf{N}_{\mathbf{C}}$ & $\mathbf{N}_{\mathbf{q}}$ & $\mathbf{N}_{r}$ & $\begin{array}{l}\mathrm{qs}_{\mathrm{s}} \\
\left(\mathrm{kg} / \mathrm{cm}^{2}\right)\end{array}$ \\
\hline Neogi Nagar & 15.5 & 73.82 & 0.3 & 1.5 & 1.5 & 1 & 15.6 & 10.2 & 9.73 & 2.76 & 1.25 & 75.23 \\
\hline $\begin{array}{l}\text { School at } \\
\text { Campal }\end{array}$ & 15.5 & 73.82 & 1.3 & 1.5 & 1.5 & 1.5 & 0 & 31.2 & 34 & 22 & 28.6 & 40.16 \\
\hline $\begin{array}{l}\text { Panaji } \\
\text { (Hotel } \\
\text { Nova) }\end{array}$ & 15.5 & 73.82 & 0.87 & 1.5 & 1.5 & 1.3 & 16.7 & 25.6 & 21.4 & 11.2 & 11.5 & 236.84 \\
\hline Mapusa & 15.61 & 73.81 & 0.43 & 1.5 & 1.5 & 1.5 & 22.6 & 14.9 & 10.9 & 3.91 & 2.62 & 130.59 \\
\hline $\begin{array}{l}\text { Ucassaim, } \\
\text { Mapusa }\end{array}$ & 15.58 & 73.84 & 1.1 & 1.5 & 1.5 & 1.7 & 0 & 23 & 18.4 & 8.95 & 8.68 & 16.09 \\
\hline $\begin{array}{l}\text { M/S Aces } \\
\text { Patto, Panaji }\end{array}$ & 15.5 & 73.83 & 0.45 & 1.5 & 1.5 & 1.2 & 4.7 & 7.4 & 7.38 & 2 & 0.81 & 19.64 \\
\hline $\begin{array}{l}\text { Ravindra } \\
\text { Bhavan, } \\
\text { Baina, } \\
\text { Vasco }\end{array}$ & 15.39 & 73.97 & 3 & 1.5 & 1.5 & 1.5 & 0 & 36.1 & 52.5 & 40.1 & 61.2 & 99.81 \\
\hline $\begin{array}{l}\text { Bridge at } \\
\text { Sonarbag } \\
\text { Usgao, } \\
\text { Vaghurme }\end{array}$ & 15.44 & 74.05 & 20.35 & 1.5 & 1.5 & 1.7 & 15 & 23 & 18.4 & 8.95 & 8.68 & 1330.41 \\
\hline
\end{tabular}

Table 3 SBC calculation table (by $\mathrm{N}$ value) (for 3.0m)

\begin{tabular}{|c|c|c|c|c|c|c|c|c|c|c|c|c|c|}
\hline Place & Lat & Long & $\begin{array}{l}\text { SPT } \\
(\mathbf{N})\end{array}$ & $\begin{array}{l}\mathbf{W T} \\
(\mathbf{m}) \\
\left(\mathbf{D}_{\mathbf{t}}\right)\end{array}$ & B & D & $\Upsilon_{d}$ & $\begin{array}{l}\mathrm{C} \\
(\mathrm{kN} \\
\left./ \mathrm{m}^{2}\right)\end{array}$ & $\begin{array}{l}\varphi \\
\left({ }^{\circ}\right)\end{array}$ & $\mathbf{N}_{\mathbf{C}}$ & $\mathbf{N}_{\mathbf{q}}$ & $\mathbf{N}_{\mathbf{r}}$ & $\begin{array}{l}\mathrm{q}_{\mathrm{s}} \\
\left(\mathrm{kN} / \mathrm{m}^{2}\right)\end{array}$ \\
\hline Neogi Nagar & 15.498 & 73.821 & 2 & 0.3 & $\begin{array}{r}1 . \\
5\end{array}$ & 3 & 1.03 & 0 & 28 & 26.4 & 15.3 & 17.79 & 72.07 \\
\hline $\begin{array}{l}\text { School at } \\
\text { Campal }\end{array}$ & 15.495 & 73.819 & 13 & 1.3 & $\begin{array}{r}1 . \\
5\end{array}$ & 3 & 1.53 & 0 & 31 & 33.3 & 21.4 & 27.52 & 190.52 \\
\hline $\begin{array}{l}\text { Tonca, Panaji } \\
\text { (Prudential } \\
\text { groups) }\end{array}$ & 15.494 & 73.825 & 15 & $\begin{array}{r}28.5 \\
4\end{array}$ & $\begin{array}{r}1 . \\
5\end{array}$ & 3 & 1.64 & 0 & 32 & 36.5 & 24.4 & 32.65 & 1508.92 \\
\hline $\begin{array}{l}\text { Panaji (Hotel } \\
\text { Nova) }\end{array}$ & 15.498 & 73.823 & 4 & 0.87 & $\begin{array}{r}1 . \\
5\end{array}$ & 3 & 1.34 & 0 & 29 & 28.3 & 16.9 & 20.09 & 117.78 \\
\hline
\end{tabular}


International Journal of Engineering Applied Sciences and Technology, 2020

Vol. 4, Issue 10, ISSN No. 2455-2143, Pages 186-195

Published Online February 2020 in IJEAST (http://www.ijeast.com)

\begin{tabular}{|l|r|r|r|r|r|r|r|r|r|r|r|r|r|}
$\begin{array}{l}\text { Panaji (Vistar } \\
\text { motors } \\
\text { portais) }\end{array}$ & 15.495 & 73.825 & 1 & 1.3 & 5 & 3 & 1.52 & 0 & 28 & 26.4 & 15.3 & 17.79 & 130.74 \\
\hline Mapusa & 15.607 & 73.805 & 17 & 0.43 & 5 & 3 & 1.47 & 0 & 32 & 36.5 & 24.4 & 32.65 & 174.84 \\
\hline $\begin{array}{l}\text { Ucassaim, } \\
\text { Mapusa }\end{array}$ & 15.58 & 73.842 & 50 & 1.1 & 5 & 3 & 1.66 & 0 & 41 & 88 & 78.3 & 141.9 & 813.59 \\
\hline $\begin{array}{l}\text { Dry dock, } \\
\text { Bainguinim, } \\
\text { Old Goa }\end{array}$ & 15.455 & 73.814 & 1 & 1 & 5 & 3 & 1.46 & 0 & 28 & 26.4 & 15.3 & 17.79 & 118.55 \\
\hline $\begin{array}{l}\text { M/S } \\
\begin{array}{l}\text { Parsvnath } \\
\text { developers }\end{array}\end{array}$ & 15.495 & 73.824 & 1 & 0.8 & 5 & 3 & 1.5 & 0 & 28 & 26.4 & 15.3 & 17.79 & 116.99 \\
\hline $\begin{array}{l}\text { Ltd. Patto, } \\
\text { Panaji }\end{array}$
\end{tabular}

Table 4 SBC calculation table (by $C \& \varphi$ value) (for 3.0m)

\begin{tabular}{|c|c|c|c|c|c|c|c|c|c|c|c|c|}
\hline Place & Lat & Long & $\begin{array}{l}\text { WT } \\
(\mathbf{m})( \\
\left.\mathbf{D}_{\mathbf{t}}\right)\end{array}$ & B & D & $\Upsilon_{d}$ & $\begin{array}{l}\mathrm{C} \\
\left(\mathbf{k N} / \mathbf{m}^{2}\right)\end{array}$ & $\varphi\left({ }^{\circ}\right)$ & $\mathbf{N}_{\mathbf{C}}$ & $\mathbf{N}_{q}$ & $\mathbf{N}_{\mathbf{r}}$ & $\begin{array}{l}\mathbf{q}_{\mathrm{s}} \\
\left(\mathbf{k N} / \mathbf{m}^{2}\right)\end{array}$ \\
\hline $\begin{array}{l}\text { Neogi } \\
\text { Nagar }\end{array}$ & 15.498 & 73.821 & 0.3 & 1.5 & 3 & 1.03 & 15.6 & 10.2 & 9.73 & 2.76 & 1.25 & 77.92 \\
\hline $\begin{array}{l}\text { School at } \\
\text { Campal }\end{array}$ & 15.495 & 73.819 & 1.3 & 1.5 & 3 & 1.53 & 0 & 31.2 & 33.97 & 21.97 & 28.55 & 68.51 \\
\hline $\begin{array}{l}\text { Panaji } \\
\text { (Hotel } \\
\text { Nova) }\end{array}$ & 15.498 & 73.823 & 0.87 & 1.5 & 3 & 1.34 & 16.65 & 25.6 & 21.42 & 11.17 & 11.53 & 248.79 \\
\hline Mapusa & 15.607 & 73.805 & 0.43 & 1.5 & 3 & 1.47 & 22.6 & 14.9 & 10.92 & 3.91 & 2.62 & 135.57 \\
\hline $\begin{array}{l}\text { Ucassaim, } \\
\text { Mapusa }\end{array}$ & 15.58 & 73.842 & 1.1 & 1.5 & 3 & 1.66 & 0 & 23 & 18.36 & 8.95 & 8.68 & 28.55 \\
\hline $\begin{array}{l}\text { M/S Aces } \\
\text { Patto, } \\
\text { Panaji }\end{array}$ & 15.497 & 73.825 & 0.45 & 1.5 & 3 & 1.15 & 4.7 & 7.4 & 7.38 & 2 & 0.81 & 22.10 \\
\hline $\begin{array}{l}\text { Ravindra } \\
\text { Bhavan, }\end{array}$ & 15.391 & 73.969 & 3 & 1.5 & 3 & 1.46 & 0 & 36.1 & 52.54 & 40.09 & 61.23 & 166.13 \\
\hline
\end{tabular}


International Journal of Engineering Applied Sciences and Technology, 2020

Vol. 4, Issue 10, ISSN No. 2455-2143, Pages 186-195

Published Online February 2020 in IJEAST (http://www.ijeast.com)

\begin{tabular}{|l|r|r|r|r|r|r|r|r|r|r|} 
Baina, \\
Vasco
\end{tabular}

\section{RESULTS}

Map 1 shows the SBC ( $N$ values) of soil across the state of Goa generated using table 1. Map 2 shows the SBC (C \& $\varphi$ values) of soil across the state of Goa generated using table 2. Map 3 shows the SBC (N values) of soil across the state of Goa generated using table 3. Map 4 shows the SBC (C \& $\varphi$ values) of soil across the state of Goa generated using table 4 . 
International Journal of Engineering Applied Sciences and Technology, 2020

Vol. 4, Issue 10, ISSN No. 2455-2143, Pages 186-195

Published Online February 2020 in IJEAST (http://www.ijeast.com)

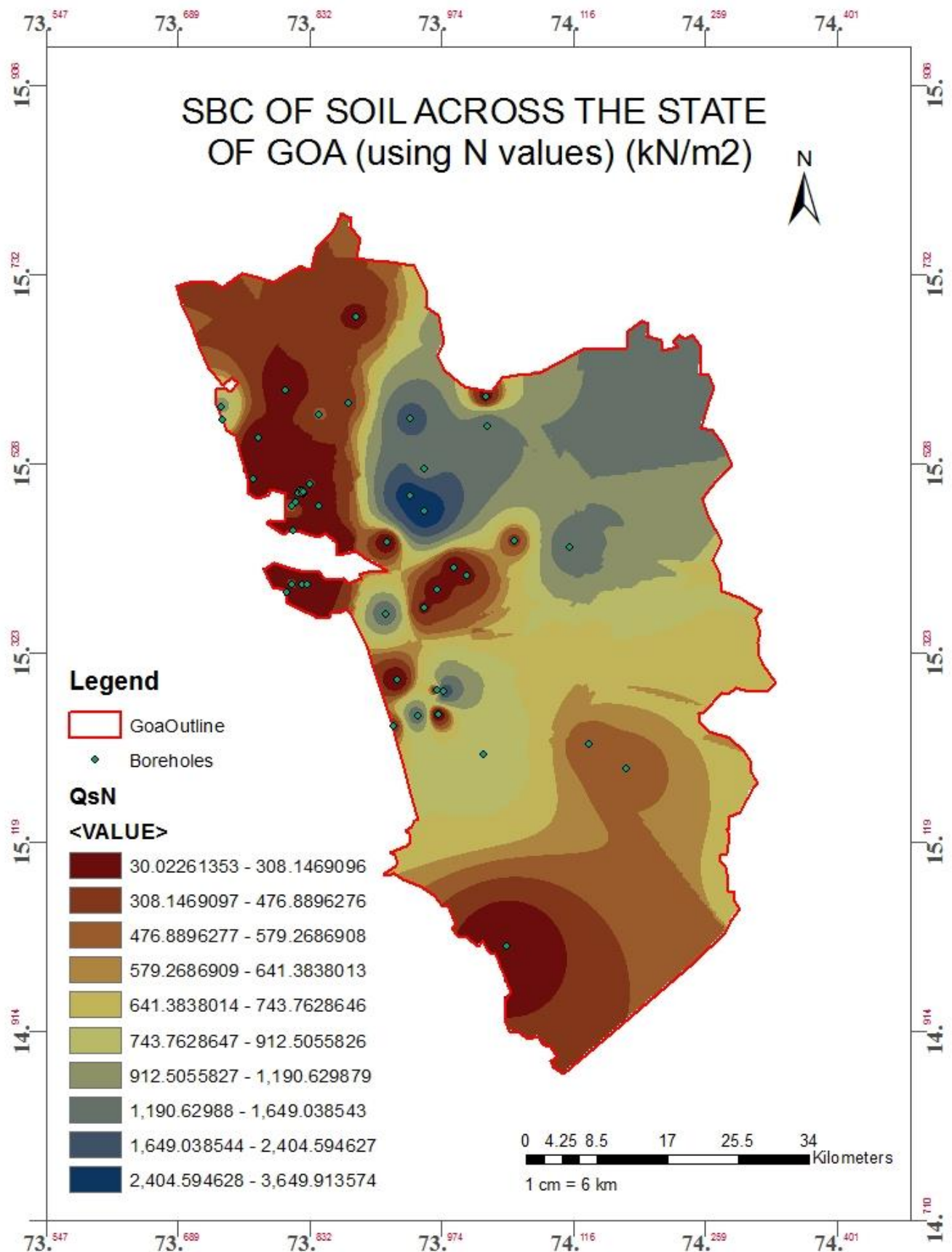

Map 1 SBC values of Goa at depth $1.5 \mathrm{~m}$ 


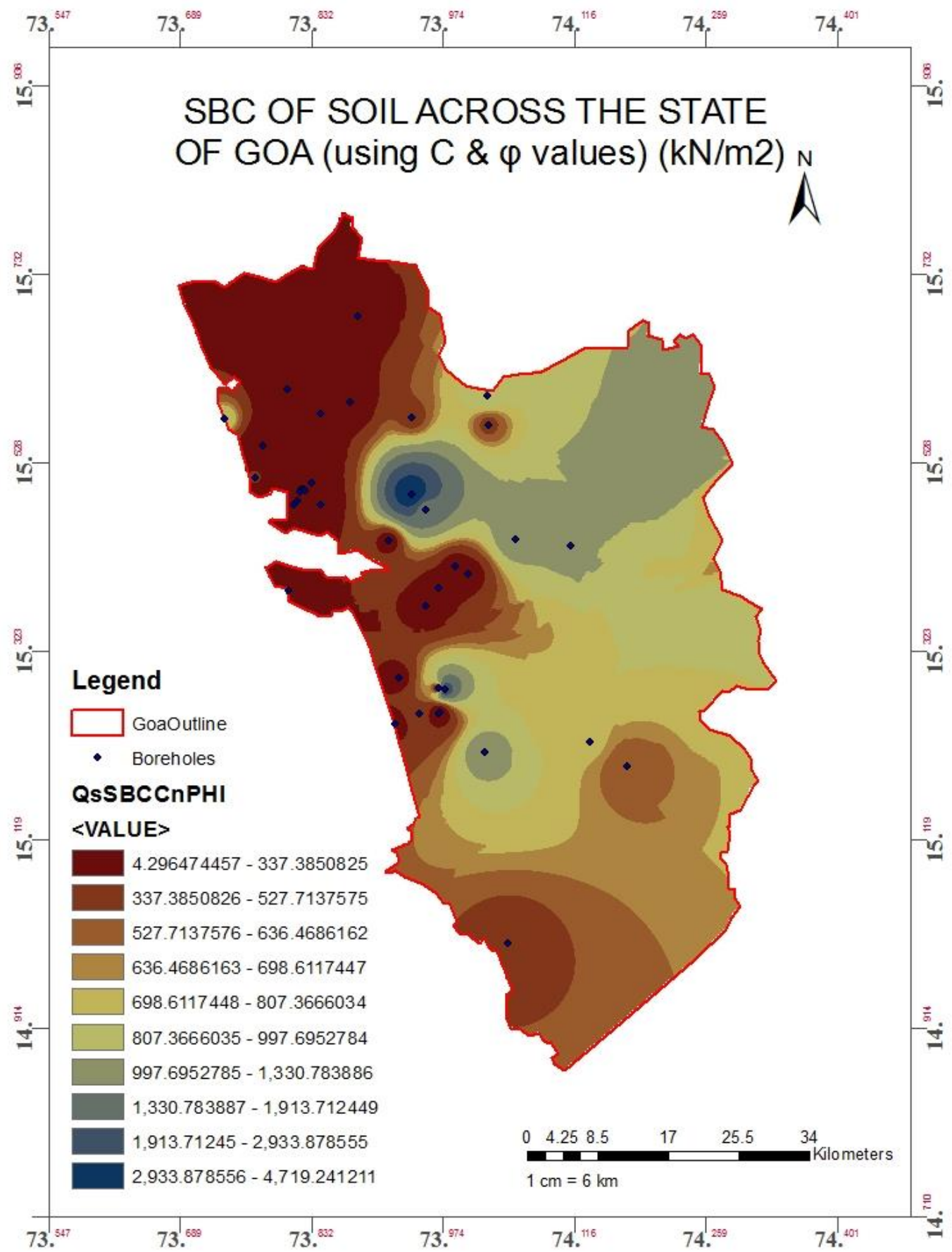

Map 2 SBC values of Goa at depth $1.5 \mathrm{~m}$ 
International Journal of Engineering Applied Sciences and Technology, 2020

Vol. 4, Issue 10, ISSN No. 2455-2143, Pages 186-195

Published Online February 2020 in IJEAST (http://www.ijeast.com)

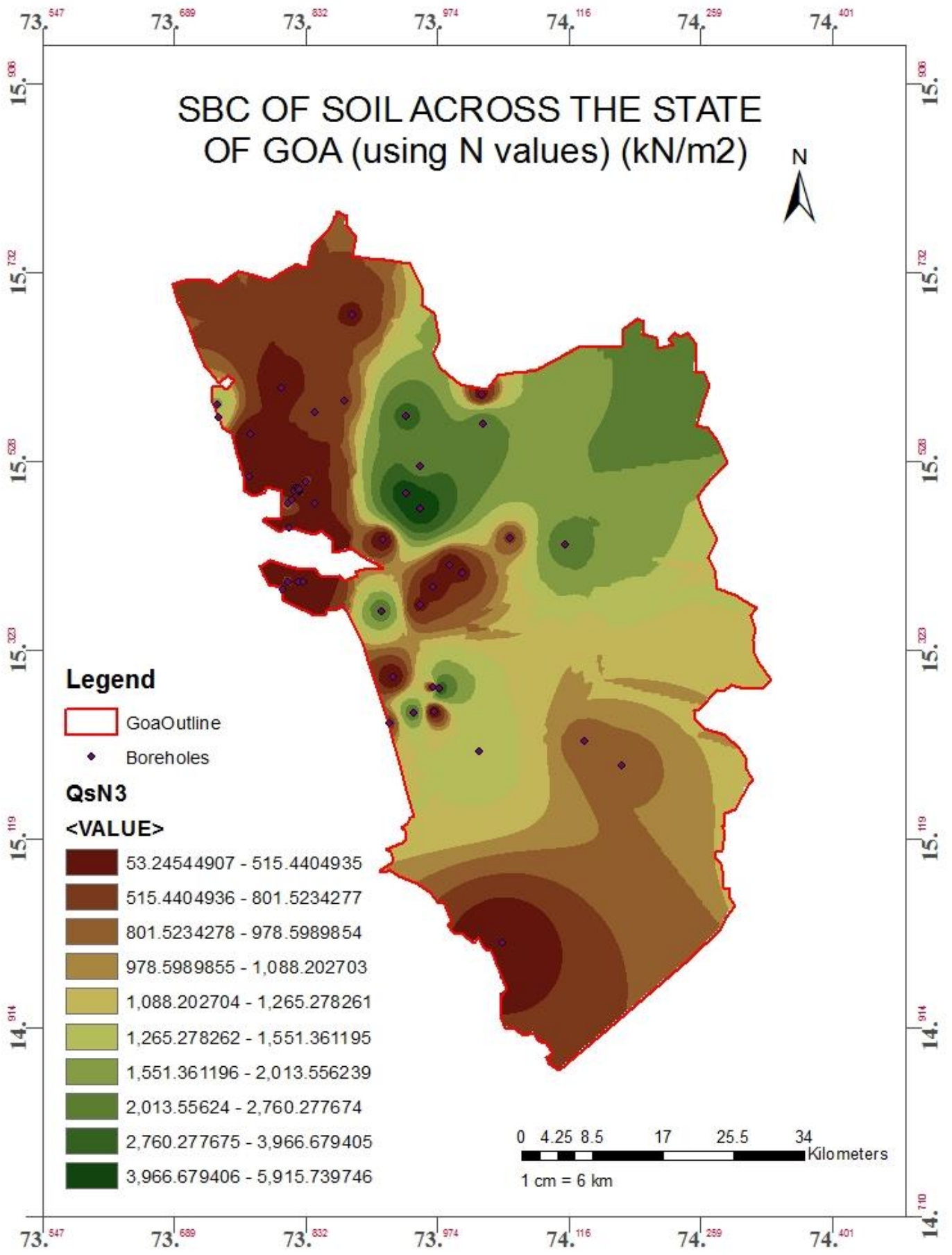

Map 3 SBC values of Goa at depth 3.0m 
International Journal of Engineering Applied Sciences and Technology, 2020

Vol. 4, Issue 10, ISSN No. 2455-2143, Pages 186-195

Published Online February 2020 in IJEAST (http://www.ijeast.com)

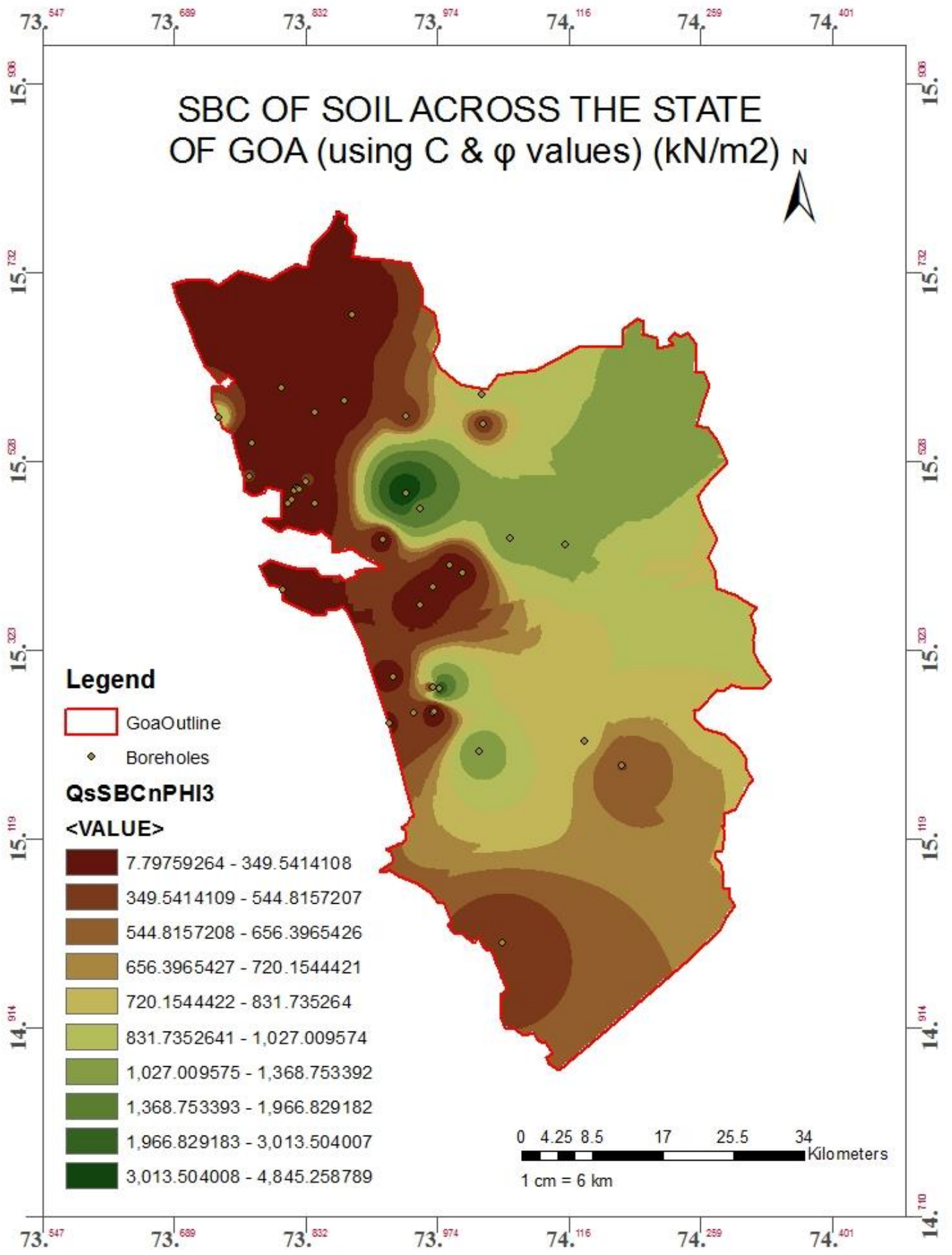

Map 4 SBC values of Goa at depth 3.0m 


\section{International Journal of Engineering Applied Sciences and Technology, 2020 Vol. 4, Issue 10, ISSN No. 2455-2143, Pages 186-195 \\ Published Online February 2020 in IJEAST (http://www.ijeast.com)}

\section{CONCLUSION}

Maps were created by using ArcGIS software. The Goa shapefiles were digitized in the software for this work. Polygon type shapefile was used to digitize and create the boundary line of Goa. Boreholes were collected from different agencies. The necessary data was extracted from the boreholes and a database was created, to input it in the software for creating different maps. The SBC calculated from $\mathrm{N}$ values vary from $30-4000 \mathrm{kN} / \mathrm{sq} . \mathrm{m}$. at $1.5 \mathrm{~m}$ depth and 50 $6000 \mathrm{kN} / \mathrm{sq} . \mathrm{m}$. at $3.0 \mathrm{~m}$ depth. The SBC calculated from $C \& \varphi$ values vary from $10-4700 \mathrm{kN} / \mathrm{sq} . \mathrm{m}$. at $1.5 \mathrm{~m}$ depth and $10-3200 \mathrm{kN} / \mathrm{sq} . \mathrm{m}$. at $3.0 \mathrm{~m}$ depth. Based on the study conducted, it can be concluded that the effective use of this maps generated can be done by contractors and engineers for preliminary idea of soil at construction site.

\section{LIMITATIONS}

It is the duty of the authors to state the limitation of the maps generated in this study. The maps are based on a data of 200 boreholes, hence entire area of the state is not equally represented in terms of borelogs density.

\section{REFERENCES}

1. Ihrke C, (2012) "Database Management and Spatial Interpolation of Geologic Boring Logs Using GIS at the Kalmar Landfill Rochester".

2. IS: 6403 (1981) CODE OF PRACTICE FOR DETERMINATION OF BEARING CAPACITY OF SHALLOW FOUNDATIONS.

3. Jumikis, A.R. (1965). Soil Mechanics, An EastWest Edition, D. Van Nostrand Company.

4. Kulkarni, M.N. (2003). Global Positioning System and its Application, CEP Training Course, IIT Bombay, pp. 1-15.

5. Mary Labib \& Amani Nashed, (2013) "GIS and geotechnical mapping of expansive soil in Toshka region", Ain Shams Engineering Journal 4, 423-433.

6. Md. Marufur Rahman \& A. Q. M. Mahbub, (2012) "Lithological Study and Mapping of Barind Tract Using Borehole Log Data with GIS: In the Context of Tanore Upazila", Journal of Geographic Information System, 4, 349-357.

7. Mhaske S. Y., \& Deepankar Choudhury, (2009) "APPLICATION OF GIS-GPS FOR MAPPING SOIL INDEX PROPERTIES”, IGC vol.2, Guntur, INDIA.

8. Mu Huang, Dapeng Li1, Xianyong Han, (March 2014) "A Stratigraphic Modeling Method based on Borehole Data", IJCSI International Journal of Computer Science Issues, Vol. 11, Issue 2, No 1 .

9. M.L.Manchanda, M.Kudrat \& A.K.Tiwari, (2002) "Soil survey and mapping using remote sensing", International Society for Tropical Ecology.

10. Phadake, V.R. and Jain, R.K. (1998). Geotechnical Engineering, Nirali Prakashan

11. Punmia B. C. , Ashok Kumar Jain \& Arun Kumar Jain, (2005) "A textbook of Soil Engineering", $16^{\text {th }}$ edition.

12. Ramamurthy, T.N. and Sitharam, T. G. (2005). Geotechnical Engineering [Soil Mechanics], S. Chand $1^{\text {st }}$ edition.

13. Rimjhim K. , Borah Sushmita \& Chetia Malaya, (28th September 2013) "Application of Remote Sensing in Soil Mapping: A Review", North East Students Geo-Congress on Advances in Geotechnical Engineering (NES Geo-Congress 2013), Guwahati.

14. Swaroopa Sail, Glancia Fernandes, Akil Inamdar, Manjunath Hiremath, Mohammed Ashraf Hullati, Santosh Kunnur, Subash Goudar, (2017) "Application of GIS In Soil Mapping”, International Journal of Engineering Research in Mechanical and Civil Engineering (IJERMCE) Special Issue National Conference on Advances in Civil and Mechanical Engineering (NCACME17).

15. Terzaghi, K. and Peck R.B. (1967) "Soil mechanics in engineering practice", 2nd edn. Wiley, New York.

16. T.G.Sitharam, P.Anbazhagan, G.U.Mahesh, K.Bharathi \& P.Nischala Reddy, (September 23-24, 2005) "SEISMIC HAZARD STUDIES USING GEOTECHNICAL BOREHOLE DATA AND GIS", Symposium on Seismic Hazard Analysis and Microzonation, Roorkee.

17. Venkatachalam, P. (2006). DST-NRDMS Sponsored Training Programme: Geospatial Technologies and Applications: Principle of GIS, GRAM++ GIS Package Development and Applications, CSRE, IIT Bombay, Mumbai. 